with psychiatric comorbidities, particularly those with depression, dementia and intellectual disability. Better optimisation of facilities and a more personalised approach to patients with $\mathrm{CPC}$ are required to improve inpatient outcomes and resource allocation.

\section{Impairments in theory of mind following traumatic brain injury: a systematic review}

\section{Radhika Chikramane}

Westmead Hospital

doi: 10.1192/bjo.2021.648

Aims. To assess the nature and extent of Theory of Mind (ToM) impairments post-TBI.

Method. Electronic databases searches included PubMed/ MEDLINE, PubMed Central, Scopus, PsychArticles, PsychINFO, Web of Science, ProQuest Central, and Wiley Online Library databases. Only studies conducted on adult patients with TBI compared with healthy controls published in English in peer-reviewed journals were considered. Reference lists were manually checked for additional studies. 19 studies were identified.

Result. Marked moderate-to-severe ToM deficits in adults post-TBI were observed across all severities of injury and chronicity. ToM deficits were documented across tasks and reflected a hierarchy where performance worsened significantly as tasks progressed in complexity. Despite supportive factors, certain aspects of ToM impairment, such as ability to detect and interpret nonliteral speech and judge appropriateness of context remained affected in the subjects.

Conclusion. ToM deficits represent a robust finding in adults with TBI. The chronicity of TBI requires a long-term view and is complicated by the fact that ToM deficits are invisible and difficult to understand. Perceptive-taking deficits faced by TBI sufferers has bio-socio-economic implications. This review also discusses implications for basic and clinical neuropsychology and rehabilitation efforts. Further research is needed, particularly in the form of large, longitudinal studies that mimic day-to-day interactions, to inform/support rehabilitation programs.

\section{Predictors of cognitive, behavioural and academic difficulties in NF1}

Kavitha Chinnappa Ramamurthy ${ }^{1 *}$, Marie-Maude Geoffray ${ }^{2}$, Louise Robinson $^{1}$, Lauren Manderson ${ }^{1}$, Julieta O'Flaherty ${ }^{1}$, Annukka Lehtonen ${ }^{1}$, Grace Vassallo ${ }^{1}$, Shruti Garg ${ }^{3}$ and Jonathan Green ${ }^{4}$

${ }^{1}$ Department of Child and Adolescent Psychiatry ,Manchester University Foundation Trust; ${ }^{2}$ Centre hospitalier le Vinatier;

${ }^{3}$ Manchester Centre for Genomic Medicine, Manchester University NHS Foundation Trust, Manchester Academic Health Sciences Centre, Department of Child and Adolescent Psychiatry, Manchester University Foundation Trust and ${ }^{4}$ Division of Neuroscience and Experimental Psychology, Faculty of Biological Medical \& Health Sciences, University of Manchester, Manchester Academic Health Sciences Centre, Department of Child and Adolescent Psychiatry, Manchester University Foundation Trust

${ }^{\star}$ Corresponding author.

doi: 10.1192/bjo.2021.649

Aims. The aim of this study is to systematically investigate the demographic and disease predictors of cognitive and behavioural phenotype in the largest cohort of children with NF1 published to date. Based on previously published research, we examine the potential role of demographic predictors such as age, sex, SES, parental NF1 status as well as the neurological complications such as epilepsy and brain tumours in NF1 associated cognitive/ behavioural impairments.

Method. In this cross-sectional study design, participant data were drawn from two large databases which included (i) A clinical database of all patients with NF1 seen in a clinical psychological service from 2010 to 2019 and (ii) A research dataset from two previously published studies $(2,8)$. The complex National NF1 service based within Manchester regional genetic services is set up for individuals with complex NF1 (https://www.mangen.co.uk/healthcare-professionals/clinical-genomic-services/nf1/) in the North of the UK. Children were referred to the psychological services by NF1 clinicians if psychological assessment was warranted based on parental reports. In order to reduce clinic referral bias, the clinical sample was supplemented by including participants that were seen solely for the purposes of research studies within our centre.

Result. Relative to population norms, $90 \%$ of the NF1 sample demonstrated significantly lower scores in at least one cognitive or behavioral domain. Family history of NF1 and lower SES were independently associated with poorer cognitive, behavioral and academic outcomes. Neurological problems such as epilepsy and hydrocephalus were associated with lower IQ and academic skills. Conclusion. Cognitive and behavioural phenotypes commonly emerge via a complex interplay between genes and environmental factors, and this is true also of a monogenic condition such as NF1. Early interventions and remedial education may be targeted to risk groups such those with familial NF1, families with lower SES and those with associated neurological comorbidities.

\section{Investigating the association between depressive disorders and cerebral haemodynamics}

Tamara Chithiramohan ${ }^{1 \star}$, Jvalant Parekh ${ }^{2}$, Lucy Beishon ${ }^{1}$, Golo Kronenberg ${ }^{1}$, Victoria Haunton ${ }^{1}$, Jatinder Minhas ${ }^{1}$, Thompson Robinson ${ }^{1}$ and Ronney Panerai ${ }^{1}$

${ }^{1}$ University of Leicester, Department of Cardiovascular Sciences and ${ }^{2}$ University Hospitals of Leicester

${ }^{*}$ Corresponding author.

doi: $10.1192 /$ bjo.2021.650

Aims. Alterations in cerebral blood flow (CBF) may contribute to the development of depression, and serve as a novel biomarker. The aim of this review is to summarise and synthesise the available evidence on alterations in cerebral haemodynamics in depressive disorders relative to healthy control populations.

Method. MEDLINE (1946- present), EMBASE (1947- present), Web of Science (1970-present), PsycINFO (1984-present), CINAHL (1976-present) and CENTRAL were searched using a predefined search strategy. Studies which compared the cerebral haemodynamics of adult patients ( $>18$ years old) with depressive disorders against healthy controls (HC), by any imagining modality, were included. Studies with varying severity and chronicity of depressive disorder were included. A meta-analysis was conducted in four groups: 1) CBF ( $\mathrm{ml} / \mathrm{min} / 100 \mathrm{~g}$ ) 2) Cerebral blood flow velocity (CBFv) (cm/s) 3) Combined $\mathrm{CBF}$ and $\mathrm{CBFv} 4)$ Ratio of uptake of radiotracer. A random effects model was used and heterogeneity and publication bias were assessed. Data are presented as mean difference (MD) or standardised mean difference (SMD) and 95\% confidence interval (95\% CI). A narrative synthesis of the remaining studies was performed.

Result. 87 studies met the inclusion criteria. CBF ( $\mathrm{ml} / \mathrm{min} / 100 \mathrm{~g})$ was significantly reduced in patients with depression compared to 